\title{
Crisis gelásticas secundarias a hamartoma hipotalámico
}

\section{Gelastic seizures due to hypothalamic hamartoma}

\section{David Islas García, ${ }^{\star}$ Jorge Alderete Berzaba, ${ }^{\ddagger}$ Claudia Vannesa Quiróz Serna, ${ }^{\S}$ Rilma Nelly Perera Canul§}

Citar como: Islas GD, Alderete BJ, Quiróz SCV, Perera CRN. Crisis gelásticas secundarias a hamartoma hipotalámico. An Med (Mex). 2020; 65 (3): 233-238. https://dx.doi.org/10.35366/95681

\section{RESUMEN}

Los hamartomas hipotalámicos son malformaciones originadas en el tuber cinereum e hipotálamo inferior. Se trata de una condición poco frecuente con una prevalencia estimada de uno a dos casos por cada 100,000 habitantes. La presentación clínica clásica se describe como una tríada que incluye crisis gelásticas, retraso en el desarrollo y pubertad precoz central. Sin embargo, se ha descrito que los hamartomas hipotalámicos tienen un amplio espectro en su presentación clínica y electroencefalográfica, por lo que su diagnóstico puede ser todo un desafío debido a que algunos síntomas pueden no manifestarse, especialmente en los casos que tienen un curso benigno. Presentamos el caso de una paciente con crisis gelásticas de inicio en la infancia temprana secundarias a un hamartoma hipotalámico, con ausencia de deterioro cognitivo y alteraciones endocrinológicas, por lo que el diagnóstico se realizó hasta la adolescencia debido al poco impacto en la calidad de vida que tenían las crisis. Fue manejada quirúrgicamente mediante ablación láser. En la actualidad se encuentra libre de crisis.

Palabras clave: Hamartoma hipotalámico, crisis gelásticas, video electroencefalograma.

Nivel de evidencia: IV

\begin{abstract}
Hypothalamic hamartomas are malformations originated at the tuber cinereum and lower hypothalamus. It is a rare condition, with an estimated prevalence of 1 to 2 cases per 100,000 inhabitants. The classic clinical presentation is a triad that includes gelastic crises, developmental delay, and central precocious puberty. However, it has been seen that hypothalamic hamartomas have a broad spectrum in their clinical and electroencephalographic presentation, so their diagnosis can be quite a challenge because some symptoms may not appear, especially in cases that have a benign course. We present the case of a patient with gelastic seizures that began in early childhood originated from a hypothalamic hamartoma, with absence of cognitive impairment and endocrinological alterations, for which the diagnosis was made until adolescence due to the little impact that the seizures had on her life quality. She was surgically treated with laser ablation, currently seizure free.
\end{abstract}

Keywords: Hypothalamic hamartoma, gelastic seizures, video electro encephalogram.

Level of evidence: $I V$

\footnotetext{
* Médico Especialista en Neuropediatría. Adscrito al Servicio de Neurología Pediátrica.

₹ Médico Especialista en Neuropediatría. Subespecialidad en Neurofisiología Clínica. Adscrito al Servicio de Neurofisiología Clínica.

$\S$ Médico especialista en Medicina de Rehabilitación. Médico Residente del segundo año de la Subespecialidad en Neurofisiología Clónica.
}

Centro Médico ABC.

Recibido para publicación: 30/06/2020. Aceptado: 03/08/2020.

Correspondencia: Dr. David Islas García

Av. Carlos Graef Fernández Núm. 154 C-3, Col. Tlaxala, 05300, Alcaldía Cuajimalpa de Morelos, CDMX, Tel: 5552723424.

E-mail: drdavidislasg@gmail.com

\author{
Abreviaturas: \\ $\mathrm{HH}=$ Hamartoma hipotalámico. \\ $C G=$ Crisis gelásticas \\ $\mathrm{PPC}=$ Pubertad precoz central \\ $\mathrm{CC}=$ Crisis convulsivas. \\ $T C G=$ Tónico clónico generalizado \\ $\mathrm{RM}=$ Resonancia magnética. \\ VEEG $=$ Video electroencefalograma \\ $E E G=$ Electroencefalograma.
}




\section{INTRODUCCIÓN}

Los hamartomas hipotalámicos (HH) son malformaciones originadas en el tuber cinereum e hipotálamo inferior. ${ }^{1}$ Son considerados como parte del espectro de las heterotopias de la sustancia gris que afectan el desarrollo del hipotálamo. Están compuestos de neuronas ectópicas y tejido glial, representan una de las causas más notables de epilepsia resistente al tratamiento farmacológico. ${ }^{2}$ Se trata de una condición rara, con una prevalencia estimada de uno a dos casos por cada 100,000 habitantes. $^{3}$

La presentación clínica clásica se considera una tríada que incluye crisis gelásticas $(\mathrm{CG})$, retraso en el desarrollo y pubertad precoz central (PPC). ${ }^{4}$ Sin embargo, se ha visto que los $\mathrm{HH}$ tienen un amplio espectro en su presentación clínica, ${ }^{5,6}$ que va desde crisis convulsivas esporádicas con desarrollo cognitivo normal hasta una encefalopatía epiléptica que se asemeja a un síndrome de Lennox-Gastaut. ${ }^{7}$ También se ha reportado que los pacientes con $\mathrm{HH}$ pueden mostrar alteraciones del comportamiento como inestabilidad emocional, irritación, agitación y agresión, también pueden desarrollar trastorno de déficit de atención e hiperactividad, trastorno de conducta, problemas de aprendizaje, del lenguaje y trastorno por ansiedad. ${ }^{3,8}$

Para el diagnóstico, se debe considerar la típica presentación clínica de esta patología así como la evidencia por imagen de la lesión. Sin embargo, esto puede ser todo un desafío debido a que muchos casos pueden diagnosticarse errónea y/o tardíamen- te, ya que es posible que algunos síntomas no se manifiesten, en particular en los casos que cursan de forma más benigna. Muchos neurólogos pueden encontrarse con sólo uno o dos de estos casos en su trayectoria. ${ }^{5,9,10}$

Presentamos el caso de una niña con inicio de CG en la infancia temprana, pero que fue diagnosticada y tratada por un $\mathrm{HH}$ hasta la adolescencia debido al curso benigno de su patología.

\section{CASO CLÍNICO}

Se trata de paciente femenino de 17 años y 10 meses de edad, producto de la primera gesta, nacida vía abdominal a las 28 semanas de gestación, requiriendo hospitalización en terapia intensiva neonatal durante dos meses. Desde su egreso recibió terapia neurología de rehabilitación hasta los 12 meses de edad, con un adecuado neurodesarrollo. Menarca a los 11 años con ciclo regular $30 \times 5$, sin presencia de alteraciones ginecológicas o endocrinológicas. Desempeño escolar y deportivo sobresaliente con adecuada integración social. Sin alteraciones conductuales.

Inicia con crisis tónico clónico generalizadas (TCG) nocturnas a los cinco años de edad, con una duración de 30 a 60 segundos presentándose en promedio cada dos o tres meses. Acude a valoración médica donde se establece tratamiento antiepiléptico no especificado, el cual fue suspendido por decisión familiar. A los seis años inicia con episodios involuntarios de risa explosiva, sin predominio de horario, factores desencadenantes o asociados a eventos no
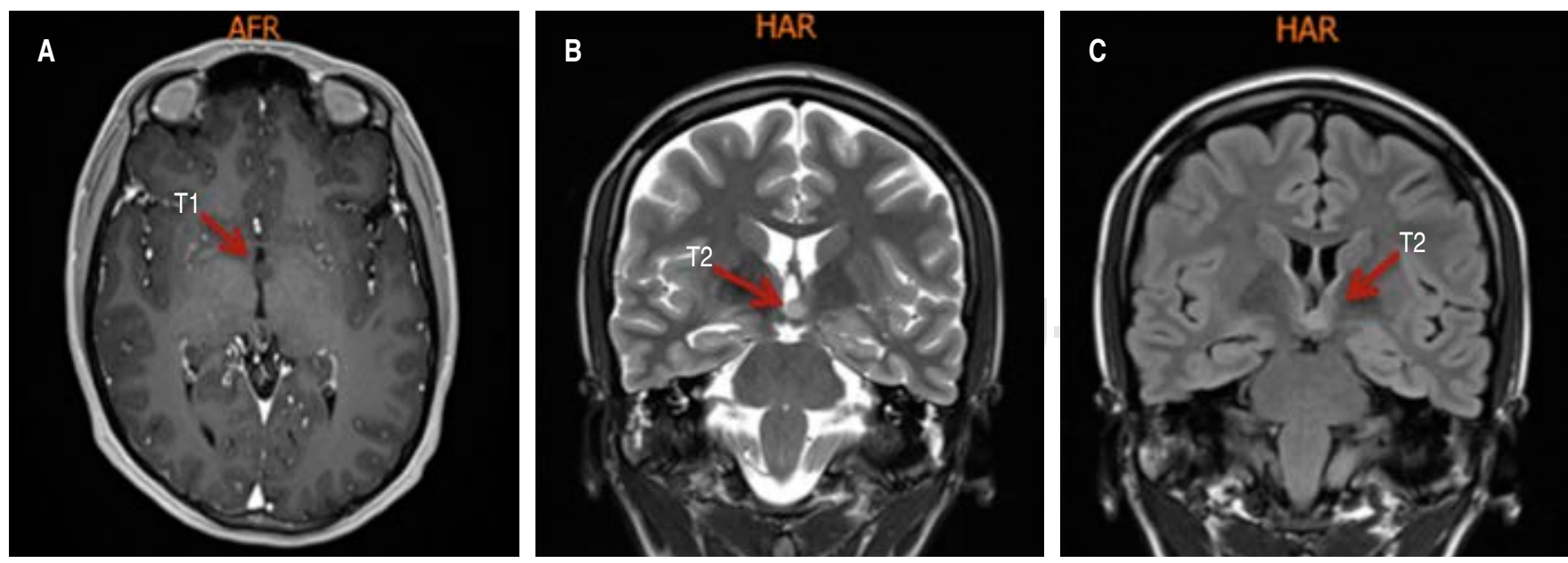

Figura 1: Resonancia magnética (RM) cerebral simple y contrastada. Imagen nodular supratentorial que desplaza caudalmente al cuerpo mamilar, ocluyendo parcialmente la luz de la parte inferior del III ventrículo izquierdo (flecha roja).

A) RM simple. Secuencia T1 corte axial. B) RM contrastada. Secuencia T2 corte coronal. C) RM simple. Secuencia T2 corte coronal. 
sardónicos, con una duración de 15 a 30 segundos y con una frecuencia de 10 a 15 eventos por mes. Se realiza vídeo electroencefalograma (VEEG) reportado como normal; sin embargo, se inicia manejo con carbamazepina, el cual se suspendió por presentar efectos secundarios (somnolencia y cefalea). Al no interferir con sus actividades de la vida diaria, escolares, deportivas o sociales, no se vuelve a buscar apoyo médico.

A los 13 años muestra diariamente exacerbación de estos episodios de risa descontrolada. La paciente identificaba el inicio de las crisis con una sensación de «felicidad» y «aumento de energía» sin desconexión con el medio. Desde los 14 años refiere que ya no recordaba los eventos nocturnos, pero sabía que había tenido un CC por tener cefalea o dolor muscular. Hace un año las crisis TCG aumentaron hasta presentarse cada 15 días, motivo por el que acude a consulta. Se encuentra exploración neurológica normal, sin déficit cognitivo, motor ni sensitivo, sin datos de focalización o lateralización, se inicia manejo a base de levetiracetam en septiembre de 2019 .

El protocolo de estudio incluyó la realización de VEEG, el cual se reportó como anormal y se captaron eventos de CG; resonancia magnética (RM) contrastada con evidencia de imagen nodular en el núcleo mamilar y la porción inferior del núcleo posterior hipotalámico izquierdo (Figura 1).

Se realiza el diagnóstico de CG secundarias a HH, se plantea manejo quirúrgico, el cual se efectuó mediante ablación láser en diciembre de 2019 en hospital externo. La cirugía se llevó a cabo sin complicaciones y sin presentar déficits endocrinológicos secundarios, reportando sólo falla de la memoria a corto plazo durante 15 días, lo cual fue relacionado al edema postquirúgico. Continuó manejo a base de levetiracetam posterior a la cirugía, el cual se retiró a finales de enero por referir cefalea y somnolencia. Actualmente la paciente se encuentra libre de crisis desde hace nueve meses y desde hace tres sin manejo antiepiléptico.

\section{DISCUSIÓN}

No existe un factor único que defina la evolución, gravedad y presentación clínica de los pacientes con un $\mathrm{HH}$, pero se ha observado que existe una relación entre el tamaño, ubicación y tipo de inserción de la lesión. ${ }^{11}$ Los $\mathrm{HH}$ que invaden al hipotálamo anterior se relacionan con alteraciones endocrinas como PPC y aquéllos que afectan la parte posterior y se encuentran en estrecha relación con los cuerpos mamilares, presentan crisis convulsivas resistentes a medica-

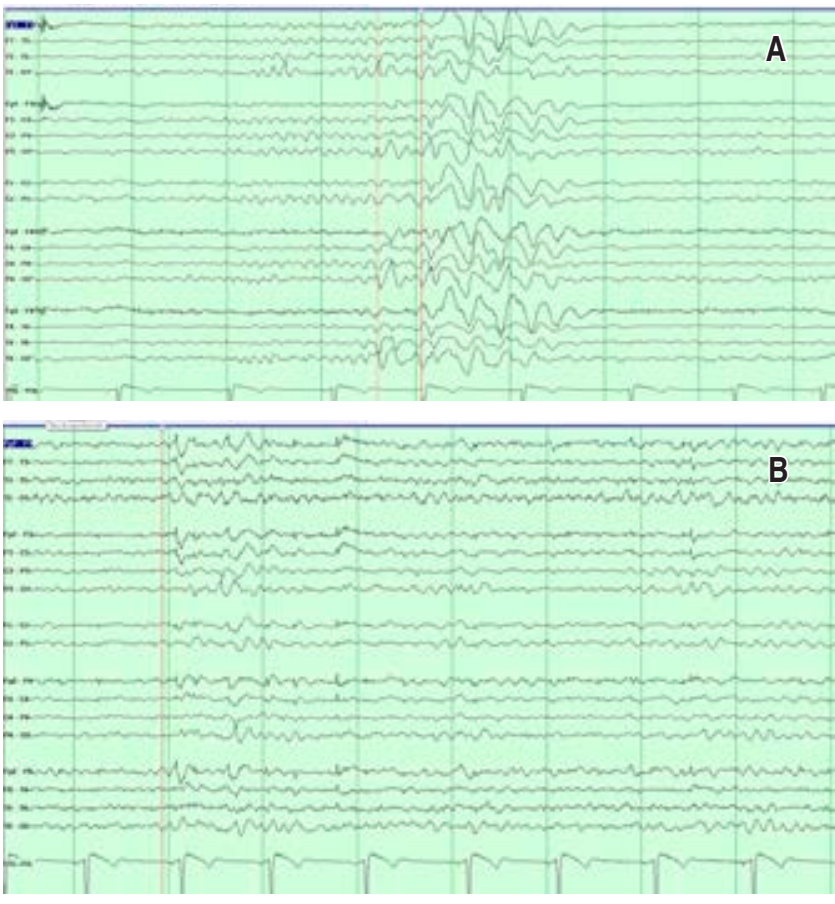

Figura 2: Registro interictal. A) Complejos de punta onda lenta generalizados. B) Puntas frontocentrales bilaterales.

mentos que generalmente comienzan en la primera infancia, como fue el caso de la paciente..$^{12-14}$

Los HH pedunculados ubicados en el hipotálamo anterior pueden causar trastornos endocrinológicos, siendo la PPC la patología más frecuente, posiblemente secundaria a una liberación prematura de hormona liberadora de gonadotropina $(\mathrm{GnRH})$ desde el hipotálamo. ${ }^{6,12,15}$

La mayoría de los casos de HH son esporádicos, pero 5\% se asocia al síndrome de Pallister Hall. ${ }^{16}$ La presentación inicial en los casos esporádicos suele ocurrir en los primeros meses de vida con CG. Al principio, las CG se caracterizan por episodios espontáneos, incontrolables y «explosivos» de risa. Los pacientes de mayor edad refieren una sensación desagradable a nivel epigástrico y una «presión» o «necesidad» por reír, pero que puede ser suprimida. ${ }^{3,7,16}$

En $50 \%$ de los casos, es posible que los pacientes progresen a un síndrome epiléptico incapacitante con presencia de múltiples tipos de crisis convulsivas (CC) refractarias a la terapia antiepiléptica, además de trastornos endocrinológicos, psiquiátricos y conductuales. . $^{6,16}$

Los niños con epilepsia secundaria a un $\mathrm{HH}$ por lo general no muestran deterioro cognitivo previo al inicio de las crisis, pero después de que éstas inician, en particular si sucede a una edad temprana, pueden 
progresivamente tener un retraso en el desarrollo y un deterioro en el comportamiento. Los pacientes que no muestran deterioro cognitivo o alteraciones en el comportamiento, presentan ataques menores con CG leves y lesiones más pequeñas. ${ }^{17}$

Se sugiere que los casos más graves que cursan con un pronunciado deterioro cognitivo son los que presentan de forma temprana las CG, ya que a mayor tiempo de evolución, la continua expansión de la actividad epiléptica del $\mathrm{HH}$ a través del tracto mamilotalámico y las áreas cerebrales asociadas produce un daño recurrente, por lo que la edad de inicio de las CG podría tener un papel predictor en la evolución de estos pacientes. ${ }^{18,19}$ En el caso de nuestra paciente, el inicio de las CG desde la infancia, aunque asociadas a crisis TCG, no repercutieron en su desarrollo ni desempeño escolar, deportivo o integración social.

El diagnóstico de los $\mathrm{HH}$ representa todo un reto. El primer contacto de los pacientes con un médico puede ser con endocrinólogos por PPC, o con neurólogos por la presencia de crisis convulsivas. Las CG, características de los $\mathrm{HH}$, pueden confundirse con crisis focales originadas en el lóbulo temporal o frontal, alteraciones conductuales, una limitada habilidad para la comunicación o simplemente pasar desapercibidas por la familia y/o el médico al no tener un impacto en su calidad de vida, por lo que el diagnóstico puede retrasarse durante algún tiempo. ${ }^{7,19}$

Se debe realizar una resonancia (RM) para confirmar o eliminar el diagnóstico de un HH. Los $\mathrm{HH}$ se expresan como una masa sólida, isointensa, no calcificada que no realza con el medio de contraste en T1 (componente longitudinal). En T2 (componente transversal) se observa una masa hiperintensa sin áreas quísticas. Una característica importante de los $\mathrm{HH}$ en la RM es que estas masas no muestran cambios a lo largo de la vida del paciente. ${ }^{9} \mathrm{La} R \mathrm{RM}$ demostró la presencia de un pequeño nódulo supratentorial en la pared izquierda del III ventrículo, diámetro anteroposterior $9.0 \mathrm{~mm}$, transversal 6.9 $\mathrm{mm}$ y cefalocaudal $6.27 \mathrm{~mm}$ que desplaza caudalmente al cuerpo mamilar de forma discreta, protruyendo en el III ventrículo ocluyendo parcialmente su luz en su parte inferior (Figura 1).

El electroencefalograma (EEG) no es una prueba específica para el diagnóstico de epilepsia secundaria a $\mathrm{HH}, 7,20$ ya que tiene una amplia variabilidad en sus resultados. Se pueden obtener registros interictales completamente normales o al contrario, con importante actividad disfuncional y epileptiforme generalizada, focal o multifocal que no correlaciona con la clínica que evidencia el paciente. Incluso, si se capta un evento, las CG pueden no mostrar patrones ictales.

Las descargas recurrentes originadas en el $\mathrm{HH}$ que se propagan a la corteza cerebral a través del tracto mamilotalámico y talamocortical, están involucradas en la generación de la actividad punta onda lenta que puede manifestarse en algunos pacientes durante los periodos interictales. ${ }^{9,21}$ Cuando hay un patrón ictal, se presenta una abrupta atenuación difusa del ritmo de fondo seguida de una breve ráfaga de actividad generalizada de polipuntas y posteriormente ráfagas de actividad rítmica rápida generalizada $(14-24 \mathrm{~Hz})$, las cuales evolucionan a una actividad de punta onda lenta. La falta de correlación eléctrica dificulta aún más el diagnóstico en pacientes con crisis sutiles. Sin embargo, el EEG permite documentar los cambios a
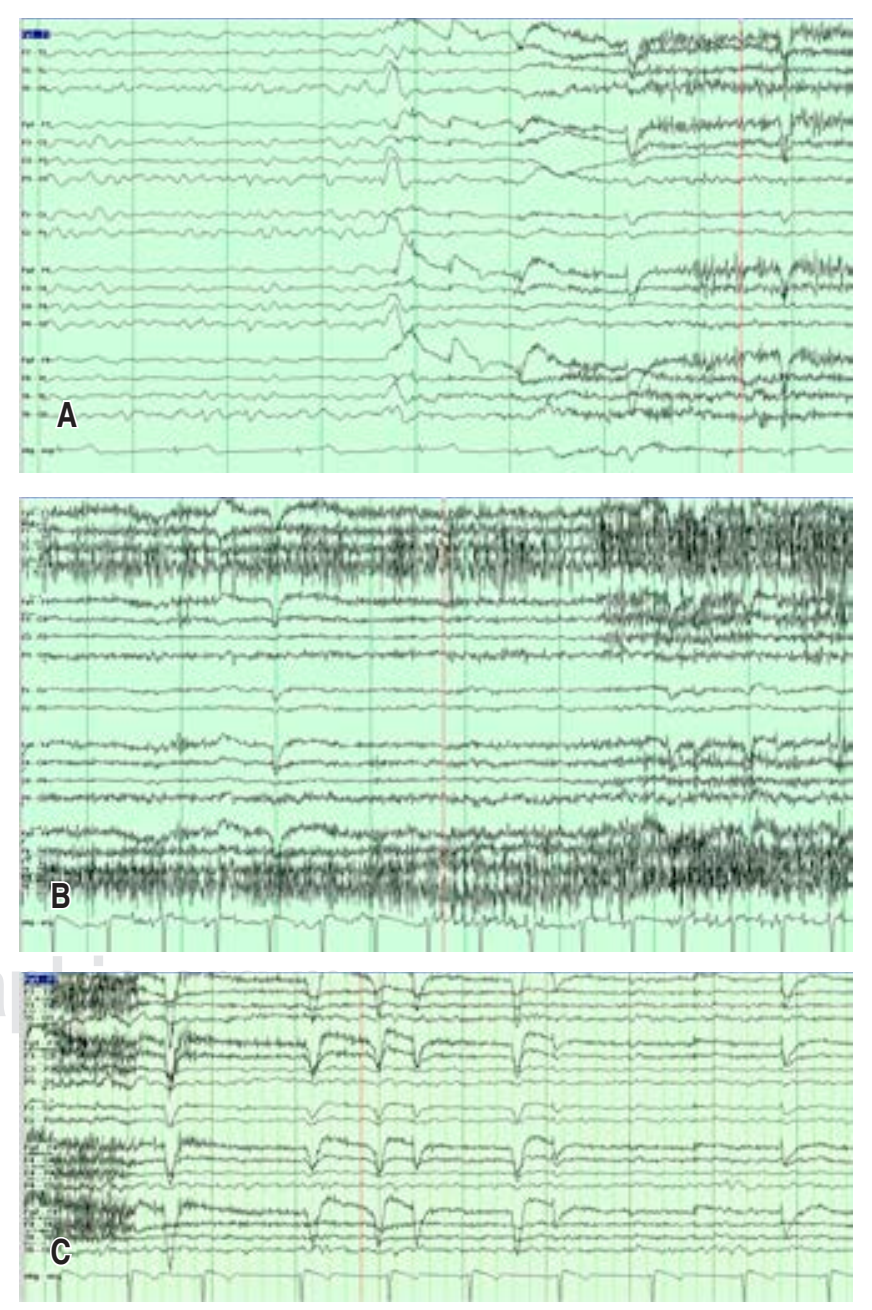

Figura 3: Registro ictal. A) Inicio de crisis con patrón evolutivo. B) Crisis gelástica. C) Fin de la crisis. 
través del tiempo y predecir el desarrollo de una encefalopatía epiléptica, por lo que forma parte de los estudios necesarios para el seguimiento de los pacientes.

El EEG de nuestra paciente presentaba un ritmo basal lento y desorganizado, brotes de puntas y ondas lentas con predominio frontocentral bilateral y complejos punta onda lenta generalizados (Figura 2). Durante el estudio se obtuvieron registros ictales que correlacionaban clínicamente con CG (Figura 3).

El tratamiento médico de las CC por lo general resulta en fracaso. Existen datos suficientes que apoyan que la medicación produce cierta diferencia en el historia natural o aporta beneficio mayor a largo plazo, ${ }^{8}$ por lo que desde 1969 se sugiere el manejo quirúrgico de los $\mathrm{HH}$ como tratamiento definitivo. ${ }^{21,22}$ Existen diversas técnicas para el manejo de estos pacientes; estudios prospectivos sugieren como primera opción la radiocirugía, con la que se obtiene un control de $60 \%$ de las crisis convulsivas. ${ }^{23,24} \mathrm{La}$ termocoagulación realizada con radiofrecuencia monopolar múltiple ha informado hasta $71 \%$ de pacientes con libertad de crisis ( $86 \%$ específicamente CG), pero con un déficit endocrino permanente en $2 \%$ de los pacientes. ${ }^{25} \mathrm{La}$ paciente cursaba con una lesión lateral con componente intraventricular y extraventricular y se le realizó una ablación láser guiada por $\mathrm{RM}$ con resultado exitoso.

\section{CONCLUSIONES}

Los HH son entidades de presentación infrecuente, por lo que se requiere un alto grado de sospecha para llegar a un adecuado diagnóstico. Existe una gran variabilidad en el curso clínico y no todos los pacientes evolucionan a un deterioro cognitivo importante, siendo las CG la única manifestación por muchos años sin afectar la calidad de vida. En otros pacientes las alteraciones endocrinológicas como la PPC puede ser la única forma de presentación.

Cuando se establece el diagnóstico de epilepsia secundaria a $\mathrm{HH}$, deberá derivarse a un centro quirúrgico especializado. Siempre debe considerarse el riesgo beneficio para el inicio de tratamiento farmacológico debido a la ya conocida falta de respuesta a los medicamentos de las CG, pero con el que otro tipo de crisis convulsivas podrían responder.

Cada caso debe individualizarse, procurando siempre una evaluación meticulosa y regular del sistema endocrino. El tipo de lesión, las manifestaciones clínicas, su impacto en la calidad de vida así como la disponibilidad de técnicas quirúrgicas en cada centro son factores que deben considerarse.
La decisión de realizar una resección o ablación temprana debe tener siempre en cuenta que el objetivo principal del tratamiento es minimizar o evitar la progresión hacia otro tipo de crisis convulsivas y por lo tanto, un deterioro cognitivo y/o conductual, factor que siempre debe plantearse al paciente y a la familia, y que se ha observado en una alta proporción de pacientes con $\mathrm{CG}$.

\section{BIBLIOGRAFÍA}

1. Tezer F, Oguz K, Saygi S. Mammillary body hamartoma in a patient with pseudotemporal lobe seizures. Acta Neurologica Belgica. 2014; 114 (3): 235-238.

2. Conde Blanco E, Anciones Martín C, Manzanares I, Gil López F, Roldán P, Donaire A, et al. Hypothalamic hamartomas in adulthood: clinical spectrum and treatment outcome-A unicenter experience. Brain Behav. 2019; 9 (11): e01412.

3. Striano S, Santulli L, Lanniciello M, Ferretti M, Romanelli $\mathrm{P}$, Striano P. The gelastic seizures-hypothalamic hamartoma syndrome: facts, hypotheses, and perspectives. Epilepsy and Behavior. 2012; 24 (1): 7-13.

4. De La Mota C, Del Valle DE, Villena A, Gero M, Del Pozo R, Rojas M. Hypothalamic hamartoma in pediatric patients: clinical characteristics, outcomes and review of the literature. Neurologia. 2012; 27 (5): 268-276.

5. Mullatti N, Selway R, Nashef L, Elwes R, Honavar M, Chandler C, Polkey C. The clinical spectrum of epilepsy in children and adults with hypothalamic hamartoma. Epilepsia. 2003; 44 (10): 1310-1319.

6. Striano S, Striano P. Clinical features and evolution of the gelastic seizures-hypothalamic hamartoma syndrome. Epilepsia. 2017; 58 (2): 12-15.

7. Cross H, Spoudeas H. Medical management and antiepileptic drugs in hypothalamic hamartoma. Epilepsia. 2017; 58: 16-21.

8. Mittal S, Mittal M, Montes J, Farmer J, Andermann F. Hypothalamic hamartomas. Part 1. Clinical, neuroimaging, and neurophysiological characteristics. Neurosurgical Focus. 2013; 34 (6): E6.

9. Wilfong A, Curry D. Hypothalamic hamartomas: optimal approach to clinical evaluation and diagnosis. Epilepsia. 2013; 54 (9): 109-114.

10. Nguyen D, Singh S, Zaatreh M. Hypothalamic hamartomas: seven cases and review of the literatura. Epilepsy and Behavior. 2003; 4 (3): 246-258.

11. Georgakoulias N, Vize C, Jenkins A, Singounas E. Hypothalamic hamartomas causing gelastic epilepsy: two cases and a review of the literature. Seizure. 1998; 7 (2): 167-171.

12. Harrison V, Oatman O, Kerrigan J. Hypothalamic hamartoma with epilepsy: Review of endocrine comorbidity. Epilepsia. 2017; 58 (2): 50-59.

13. Kerrigan J, Ng, Y, Chung S, Rekate H. The hypothalamic hamartoma: A model of subcortical epileptogenesis and encephalopathy. Seminars in Pediatric Neurology. 2005; 12 (2): 119-131.

14. Frattali C, Liow K, Craig G, Korenman L, Makhlouf F, Sato $\mathrm{S}$, Theodore W. Cognitive deficits in children with gelastic seizures and hypothalamic hamartoma. Neurology. 2001; 57 (1): $43-46$

15. Deonna T, Ziegler A. Hypothalamic hamartoma, precocious puberty and gelastic seizures: A special model of "epileptic" 
developmental disorder. Epileptic Disorders. 2000; 2 (1): 33-37.

16. Oehl B, Brandt A, Fauser S, Bast T, Trippel M, SchulzeBonhage A. Semiologic aspects of epileptic seizures in 31 patients with hypothalamic hamartoma. Epilepsia. 2010; 51 (10): 2116-2123.

17. Kerrigan J, Parsons A, Tsang C, Simeone K, Coons S, Wu J. Hypothalamic hamartoma: Neuropathology and epileptogenesis. Epilepsia. 2017; 58: 22-31.

18. Striano S, Striano P, Cirillo S, Nocerino C, Bilo L, Meo R, Briganti F. Small hypothalamic hamartomas and gelastic seizures. Epileptic Disorders. 2002; 4 (2): 129-133.

19. Arita K, Kurisu K, Kiura Y, Iida K, Otsubo H. Hypothalamic hamartoma. Neurologia Medico-Chirurgica. 2005; 45 (5): 221-231.

20. Troester M, Haine-Schlagel R, Ng Y, Chapman K, Chung S, Drees C, Kerrigan J. EEG and video-EEG seizure monitoring has limited utility in patients with hypothalamic hamartomaand epilepsy. Epilepsia. 2011; 52 (6): 1137-1143.
21. Gutierrez C, Asadi-Pooya AA, Skidmore CT, Tobochnik SD, LoPinto-Khoury C, Sperling MR. Clinical features and postoperative seizure outcome in patients with drug-resistant gelastic seizures without hypothalamic hamartoma. Epilepsy \& Behavior. 2016; 64 (Pt A): 90-93.

22. Regis J, Scavarda D, Tamura M, Nagayi M, Villeneuve N, Bartolomei F, et al. Epilepsy related to hypothalamic hamartomas: surgical management with special reference to gamma knife surgery. Childs Nerv Syst. 2006; 22: 881-895.

23. Mittal S, Mittal M, Montes J, Farmer J, Andermann F. Hypothalamic Hamartomas. Part 2. Surgical considerations and outcome. Neurosurgical Focus. 2013; 34 (6): E7.

24. Castinetti F, Brue T, Morange I, Carron R, Régis J. Gamma Knife radiosurgery for hypothalamic hamartoma preserves endocrine functions. Epilepsia. 2017; 58: 72-76.

25. Xu D, Chen T, Hlubek R, Bristol R, Smith K, Ponce F, et al. Magnetic Resonance Imaging-Guided Laser Interstitial Thermal Therapy for the Treatment of Hypothalamic Hamartomas: A Retrospective Review. Neurosurgery. 2018;83(6):1183-1192. 